\title{
Complexity based Sensing Strategy for Spectrum Sensing in Cognitive Radio Networks
}

\author{
Kewen Huang ${ }^{1}$, Yimin Liu ${ }^{1}$, Yuanquan Hong ${ }^{1}$ and Junsheng $\mathbf{M u}^{{ }^{2 *}}$ \\ ${ }^{1}$ School of physics and mechanics, Shaoguan University \\ [e-mail: 93637986@qq.com] \\ ${ }^{2}$ School of information and communication, Beijing \\ University of Posts and Telecommunications \\ [e-mai : mujs@bupt.edu.cn] \\ *Corresponding author: Junsheng Mu
}

Received November 25, 2018; revised February 9, 2019; accepted April 2, 2019; published September 30, 2019

\begin{abstract}
Spectrum sensing has attracted much attention due to its significant contribution to idle spectrum detection in Cognitive Radio Networks. However, specialized discussion is on complexity-based sensing strategy for spectrum sensing seldom considered. Motivated by this, this paper is devoted to complexity-based sensing strategy for spectrum sensing. Firstly, three efficiency functions are defined to estimate sensing efficiency of a spectrum scheme. Then a novel sensing strategy is proposed given sensing performance and computational complexity. After that, the proposed sensing strategy is extended to energy detector, Cyclostationary feature detector, covariance matrix detector and cooperative spectrum detector. The proposed sensing strategy provides a novel insight into sensing performance estimation for its consideration of both sensing capacity and sensing complexity. Simulations analyze three efficiency functions and optimal sensing strategy of energy detector, Cyclostationary feature detector and covariance matrix detector.
\end{abstract}

Keywords: Cognitive Radio Networks, spectrum sensing, sensing strategy, computational complexity 


\section{Introduction}

$\mathbf{W}_{\text {ith the rapid development of wireless communication, artificial intelligence and Internet }}$ of Things, etc., wireless business is growing explosively [1]. As a result, the traffic of radio spectrum appears to be more and more crowded [2]. However, the utilization of registered spectrum seems abnormally low according to the investigation of Federal Communications Commission (FCC) [3]. Cognitive Radio (CR) [4-5] is presented to improve spectrum utilization by reusing the idle radio spectrum of registered band when it is unoccupied. The registered users are called primary users (PU) and the unlicensed users are named secondary users (SU) in a CR [6]. The essence of a CR is to communicate with its users for SU by means of the spectrum band of PU when there is no communication for $\mathrm{PU}$ at a certain time and in a certain space. Consequently, how to obtain the band state of PU works as a significant factor for a CR. This key technology behind CR is defined as spectrum sensing (SS) [7-9] .

The main contribution of SS consists in the spectrum state judgment of observed band. Factually, SS is a kind of signal detection based on binary hypothesis [10]. In one case, both signal and noise exist. In another case, there is only noise at the receiver of a CR. Generally, three aspects are considered to estimate a SS scheme, detection probability, false alarm probability and available throughput [11]. A miss in detection will cause the interference with PU and a false alarm will reduce the spectrum efficiency [12]. The available throughput exhibits the communication capability of secondary network, which is inversely proportional to the false alarm probability under equivalent conditions [13].

Classical SS schemes include energy detector (ED) [14], Cyclostationary feature detector (CFD) [15], covariance matrix detector (CMD) [16] and cooperative spectrum sensing (CSS) [17]. ED is a widely accepted method for SS due to its low complexity and superior performance. However, it is sensitive to variational environment noise, especially in low SNR regimes. The main advantage of CFD lies in its higher sensing performance in strong noise conditions while it is at the expense of computational complexity. CMD is a blind detection scheme with no need for the prior knowledge of signal, channel and noise. Similarly, the complexity of CMD is extremely considerable compared with ED. CSS obtains the spectrum condition based on the joint judgement of multiple CR equipment placed in distinct positions. This is super helpful to solve hidden terminal problem and improves sensing performance as well.

The sensing strategy of spectrum sensing considers the primary factors that influence sensing performance and it determines the majorization direction and upper limit of sensing performance. Most alternative sensing strategy of spectrum sensing only contain part main elements of influencing sensing performance. This lowers the adaptability and robustness of cognitive system, which goes against the application and popularization of spectrum sensing in complex network environment. As a result, research on global sensing strategy that considers the whole primary factor influencing sensing performance deserves to be conducted.

Computational complexity of a scheme denotes the algorithm efficiency and implementation cost, which is a crucial factor that influences sensing performance of a CR. In consequence, sensing strategy with computational complexity is conducive to performance majorization of SS. To the best knowledge of the author, there is no related work about the discussion of complexity-based sensing strategy for SS. Motivated by this, this paper mainly discusses sensing strategy-based SS given both sensing performance and computational complexity. Firstly, three efficiency functions are defined to estimate sensing efficiency of a 
spectrum scheme from different point of view. Then a novel sensing strategy is proposed given sensing performance and computational complexity. After that the proposed sensing strategy is extended to ED, CFD, CMD and CSS. Simulations validate the reasonability and effectiveness of the proposed scheme.

The rest of this paper is organized as follows. Section 2 reviews the basic work of ED, CFD, CMD and CSS. The main contribution of this paper is shown in Section 3, which contains three efficiency functions, complexity-based sensing strategy and some application extensions. Simulation experiments and result analysis are accomplished in Section 4. Finally, Section 5 concludes this paper.

\section{Related Work}

The basic work on ED, CFD, CMD and CSS is reviewed in this section.

Assume the signal $r(t)$ at the receiver of $\mathrm{CR}$ is an additive combination of clean signal $x(t)$ and noise $w(t)$, then

$$
\begin{gathered}
r(t)=x(t)+w(t), \\
x(t)=\sum_{i=1}^{M} \phi_{i} h_{i}(t) x_{i}(t),
\end{gathered}
$$

where $x(t)$ and $w(t)$ are independent and identically distributed stationary random process with mean zero and variance $\sigma_{x}^{2}, \sigma_{w}^{2}$, respectively. $M \geq 1$ denotes the number of primary users and $\phi_{i} \in\{0,1\}$ signifies the absence or presence of the ith PU. Additionally, $h_{i}(t)$ indicates the channel gain of the $i$ th PU with mean zero and $\left|h_{i}(t)\right|$ follows Nakagami distribution.

After sampling at the rate of $f_{s}$, the received signal based on binary hypotheses can be written as

$$
\begin{aligned}
& H_{0}: r(n)=w(n) \\
& H_{1}: r(n)=\sum_{i=1}^{M} \phi_{i} h_{i}(n) X_{i}(n)+w(n),
\end{aligned}
$$

where $H_{0}$ denotes the absence of PU; $H_{1}$ means the presence of PU.

\subsection{ED}

Assume sensing duration is $\tau$ at a sensing event, then the corresponding number of samples reaches $N=\tau f_{s}$. As a result, the average energy at the receiver of CR [18] could be described as

$$
E(r)=\frac{1}{N} \sum_{n=1}^{N}|r(n)|^{2}
$$

The false alarm probability and detection probability [19] are respectively formulated as

$$
\begin{aligned}
& P_{f}=Q\left(\left(\frac{\varepsilon}{\sigma_{n}^{2}}-1\right) \sqrt{\tau f_{s}}\right), \\
& P_{d}=Q\left(\left(\frac{\varepsilon}{\sigma_{n}^{2}}-\gamma-1\right) \sqrt{\frac{\tau f_{s}}{2 \gamma+1}}\right), \\
& Q(x)=\frac{1}{\sqrt{2 \pi}} \int_{x}^{\infty} \exp \left(-\frac{t^{2}}{2}\right) d t,
\end{aligned}
$$


where $\varepsilon$ is the presupposed threshold; $\gamma=\sigma_{s}^{2} / \sigma_{n}^{2}$ represents received signal-to-noise ratio (SNR) of a CR; $Q(x)=1-\Phi(x)$ denotes complementary distribution function of standard Gaussian $\Phi(x)$.

The throughput of SU is defined as $C_{0}$ and $C_{1}$ in the absence and presence of PU, respectively. Since a length of $\tau$ period out of the total frame time $T$ is used for sensing [20], the achievable throughputs of the SUs under these scenarios are, respectively, given as

$$
\begin{aligned}
& R_{0}(\tau, \varepsilon)=C_{0} P\left(H_{0}\right)\left(1-\frac{\tau}{T}\right)\left(1-P_{f}(\tau, \varepsilon)\right), \\
& R_{1}(\tau, \varepsilon)=C_{1} P\left(H_{1}\right)\left(1-\frac{\tau}{T}\right)\left(1-P_{d}(\tau, \varepsilon)\right),
\end{aligned}
$$

where $P\left(H_{0}\right)$ and $P\left(H_{1}\right)$ respectively denote the absence and presence probability of PU in the observed bands. We assume SU could immediately stop communicating with its users $\left(C_{1} \approx 0\right)$ as soon as PU resumes the observed band in this paper. As a result, the average achievable throughput of SU [20] could be reformulated as

$$
R(\tau, \varepsilon)=C_{0} P\left(H_{0}\right)\left(1-\frac{\tau}{T}\right)\left(1-P_{f}(\tau, \varepsilon)\right)
$$

\subsection{CFD}

A continuous time random process $r(t)$ is said to be second-order Cyclostationary if its mean and autocorrelation show periodicity in time [21]. Mathematically this means that for a cyclic period $T, r(t)[21]$ can be represented as

for all $t$ and $\tau$.

$$
\begin{aligned}
& E[r(t)]=E[r(t+T)], \\
& R(r, t)=E[r(t) r(t+\tau)]=E[r(t+T) r(t+\tau+T)],
\end{aligned}
$$

The Fourier representation of (13) [22] could described as

$$
R(r, \tau)=\sum_{\alpha} R_{r}^{\alpha}(\tau) e^{j 2 \pi \alpha t} .
$$

The Fourier coefficients of (14) [23] can be expressed as

$$
R_{r}^{\alpha}(\tau)=\lim _{N \rightarrow \infty} \sum_{\tau=0}^{N-1} R(r, \tau) e^{-j 2 \pi \alpha \tau},
$$

where $\alpha$ is named as the cyclic frequency of $\mathrm{R}(r, \tau)$ and $R_{r}^{\alpha}(\tau)$ is called the cyclic autocorrelation function (CAF) [24].

After Fourier transform for CAF, the cyclic spectral density (CSD) [25] could be obtained

$$
S_{r}^{\alpha}(f)=\sum_{\tau=-\infty}^{+\infty} R_{r}^{\alpha}(\tau) e^{-j 2 \pi f \tau} .
$$

Assume that the CSD of clean signal and noise are respectively defined as $S_{x}^{\alpha}(f)$ and $S_{w}^{\alpha}(f)[26]$, then

$$
S_{r}^{\alpha}(f)=S_{x}^{\alpha}(f)+S_{w}^{\alpha}(f) .
$$

On the basis mentioned above, the false alarm probability and detection probability of CFD [27] could be expressed as

$$
\begin{gathered}
P_{f}=P\left(S_{r}^{0}(f)>0 \mid \mathrm{H}_{0}\right), \\
P_{d}=P\left(S_{r}^{0}(f)>0 \mid \mathrm{H}_{1}\right) .
\end{gathered}
$$




\subsection{CMD}

$L$ consecutive samples are considered in CMD and we define the following vectors [28]:

$$
\begin{aligned}
& r(n)=\left[\begin{array}{llll}
r(n) & r(n-1) & \ldots & r(n-L+1)
\end{array}\right]^{T}, \\
& s_{i}(n)=\left[\begin{array}{llll}
s_{i}(n) & s_{i}(n-1) & \ldots & s_{i}(n-L+1)
\end{array}\right]^{T}, \\
& n(n)=\left[\begin{array}{llll}
n(n) & n(n-1) & \ldots & n(n-L+1)
\end{array}\right]^{T} .
\end{aligned}
$$

The statistical covariance matrices of $r(n)$ could be formulated as

$$
R_{r}=E\left[r(n) r^{T}(n)\right],
$$

Let us define $\alpha_{m n}$ as the $m$ th row and $n$th of matrix $R_{r}$ then

$$
\begin{aligned}
& T_{1}=\frac{1}{L} \sum_{m=1}^{L} \sum_{n=1}^{L}\left|\alpha_{m n}\right|, \\
& T_{2}=\frac{1}{L} \sum_{n=1}^{L} \sum_{n=1}^{L}\left|\alpha_{n n}\right| .
\end{aligned}
$$

At hypothesis $H_{0}, T_{1}=0(m \neq n)$ since noise is an independent and identically distributed random process. That is, $T_{1}=T_{2}$ at hypothesis $H_{0} ; T_{1}>T_{2}$ at hypothesis $H_{1}$. Therefore, the ratio of $T_{1} / T_{2}$ is regarded as one possible scheme to compare with a presupposed threshold $\lambda_{1}$ for the presence or absence of PU [28]. However, $T_{1}=0(m \neq n)$ is always false in practice on the account of the limited samples.

Finally, the false alarm and detection probability [28] could be severally exhibited as

$$
\begin{aligned}
& P_{f a} \approx \Phi\left(\sqrt{N_{s} / 2}\left[1 / \lambda_{1}\left(1+(L-1) \sqrt{2 / N_{s} \pi}\right)-1\right]\right), \\
& P_{d}=\Phi\left(\sqrt{N_{s} / 2}\left[1 / \lambda_{1}+\lambda_{L} \gamma / \lambda_{1}(\gamma+1)-1\right]\right), \\
& \lambda_{L} \triangleq \frac{2}{L} \sum_{l=1}^{L-1}(L-l)\left|\alpha_{l}\right|, \\
& \lambda_{1}=\frac{1+(L-1) \sqrt{\frac{2}{N_{s} \pi}}}{1-\Phi^{-1}\left(1-\overline{P_{f a}}\right) \sqrt{\frac{2}{N_{s}}}} \\
& \alpha_{l}=
\end{aligned}
$$

where $N_{s}$ is the available sample number, $\Phi(x)=1-Q(x)$; $\lambda_{1}$ is the presupposed threshold for given false alarm probability; $\lambda_{L}$ denotes the overall correlation strength among the consecutive $L$ samples.

\subsection{CSS}

We assume that both detection probability and false alarm probability are identical for each node in CSS. In addition, the node number is considered as $K$. Then when AND fusion rule [29] is considered for CSS, the false alarm probability and detection probability are shown as

$$
P_{f}(N, \varepsilon)=\left(P_{f}\right)^{K},
$$




$$
P_{d}(N, \varepsilon)=\left(P_{d}\right)^{K} .
$$

If OR fusion rule [30] works in CSS, then the false alarm probability and detection probability can be given as

$$
\begin{aligned}
& P_{f}(N, \varepsilon)=1-\left(1-P_{f}\right)^{K} . \\
& P_{d}(N, \varepsilon)=1-\left(1-P_{d}\right)^{K} .
\end{aligned}
$$

When N-out-of-K fusion rule [31] is applied to CSS, the overall detection probabilities and false alarm probabilities are respectively rewritten as

$$
\begin{aligned}
& P_{d}(N, \varepsilon)=\sum_{i=N}^{K}\left(\begin{array}{c}
K \\
i
\end{array}\right) P_{d}^{i}\left(1-P_{d}\right)^{K-i}, \\
& P_{f}(N, \varepsilon)=\sum_{i=N}^{K}\left(\begin{array}{c}
K \\
i
\end{array}\right) P_{f}^{i}\left(1-P_{f}\right)^{K-i} .
\end{aligned}
$$

where $\left(\begin{array}{c}K \\ i\end{array}\right)$ is the basic combination expression and $\left(\begin{array}{c}K \\ i\end{array}\right)=C_{K}^{i}$.

\section{Main Work and Contribution}

Based on ED, CFD, CMD and CSS, a novel sensing strategy is proposed to estimate their performance given both sensing performance and computational complexity in this section. Then the sensing strategy is applied to ED, CFD, CMD and CSS for optimal performance estimation.

\subsection{Definitions}

Firstly, some indispensable definitions are provided for convenience to give the novel sensing strategy proposed in this paper.

Definition 1: For a certain detection probability $P_{d}$, if the number of the multiplication and addition operations needed is expressed as $O\left(P_{d}\right)$, we define the efficiency function of a CR to protect PU as

$$
\eta_{d}=\frac{P_{d}}{O\left(P_{d}\right)} .
$$

Definition 2: If a false alarm occurs with probability $P_{f}$ for a CR and its corresponding complexity is with $O\left(P_{f}\right)$, then we define the efficiency function of a false alarm as

$$
\eta_{f}=\frac{P_{f}}{O\left(P_{f}\right)} .
$$

Definition 3: For a certain available throughput $R(\tau, \varepsilon)$ of $\mathrm{CR}$ with sensing complexity $O(R(\tau, \varepsilon))$, the communication efficiency of secondary network is defined as

$$
\begin{aligned}
& \eta_{R}=C \frac{1-P_{f}}{O(R(\tau, \varepsilon))} . \\
& C=C_{0} P\left(H_{0}\right)\left(1-\frac{\tau}{T}\right) .
\end{aligned}
$$


Definition 4: For given $P_{d}, P_{f}$ and $R(\tau, \varepsilon)$, the overall efficiency function for a CR is given as

$$
\begin{aligned}
\eta & =\eta_{d}+\eta_{R}-\eta_{f} \\
& =\frac{P_{d}}{O\left(P_{d}\right)}+C \frac{1-P_{f}}{O(R(\tau, \varepsilon))}-\frac{P_{f}}{\mathrm{O}\left(P_{f}\right)},
\end{aligned}
$$

Note that detection probability is beneficial to secure communication of PU and available throughput indicates communication capacity of SU while false alarm probability is to the disadvantage of a CR. Consequently, the operation is described as shown in (40). Additionally, a higher $\eta_{d}$ denotes a better protection efficiency to PU while $\eta_{R}$ indicates the communication efficiency in secondary network. For convenience, $C=1$ in this paper. Consequently, (40) can be rewritten as

$$
\eta=\frac{P_{d}}{O\left(P_{d}\right)}+\frac{1-P_{f}}{O(R(\tau, \varepsilon))}-\frac{P_{f}}{\mathrm{O}\left(P_{f}\right)}
$$

\subsection{Novel sensing strategy}

In this section, given detection probability, false alarm probability, achievable throughput and computational complexity, a novel sensing strategy is proposed. It is defined as

$$
\begin{aligned}
F & =\eta_{\max } \\
& =\left[\frac{P_{d}}{O\left(P_{d}\right)}+\frac{1-P_{f}}{O(R(\tau, \varepsilon))}-\frac{P_{f}}{\mathrm{O}\left(P_{f}\right)}\right]_{\max } .
\end{aligned}
$$

Theorem 1: For ED, sensing strategy $F$ reaches maximum when

and

$$
\varepsilon=\varepsilon_{1}=\left[\frac{1}{2}+\sqrt{\frac{1}{4}+\frac{\gamma}{2}+\frac{2 \gamma+1}{\gamma \sqrt{N}} \ln 2 \sqrt{2 \gamma+1}}\right] \sigma_{n}^{2},
$$

Proof: For ED,

$$
F_{\max }=\frac{P_{d}+1-2 P_{f}}{2 N+2} \mid \varepsilon=\varepsilon_{1} .
$$

$$
O\left(P_{d}\right)=O(R(\tau, \varepsilon))=\mathrm{O}\left(P_{f}\right) \approx 2 N+2 .
$$

According to (6), (7) and (43),

$$
\frac{\partial \mathrm{F}}{\partial \varepsilon}=\frac{\partial P_{d}}{\partial \varepsilon}-2 \frac{\partial P_{f}}{\partial \varepsilon}
$$

Then

$$
\begin{aligned}
& \frac{\partial \mathrm{F}}{\partial \varepsilon} \leq 0 \Leftrightarrow \frac{\partial P_{d}}{\partial \varepsilon} / \frac{\partial P_{f} \leq}{\partial \varepsilon} \leq \\
& \Leftrightarrow \frac{\varepsilon^{2}}{\sigma_{n}^{4}}-\frac{\varepsilon}{\sigma_{n}^{2}}-\frac{\gamma}{2} \leq \frac{2 \gamma+1}{\gamma \sqrt{N}} \ln 2 \sqrt{2 \gamma+1} \\
& \Leftrightarrow \varepsilon_{\leq} \geq\left[\frac{1}{2}+\sqrt{\frac{1}{4}+\frac{\gamma}{2}+\frac{2 \gamma+1}{\gamma \sqrt{N}} \ln 2 \sqrt{2 \gamma+1}}\right] \sigma_{n}^{2} .
\end{aligned}
$$


For CFD, the proposed sensing strategy could be further described as

$$
\begin{aligned}
F & =\left[\frac{P_{d}}{O\left(P_{d}\right)}+\frac{1-P_{f}}{O(R(\tau, \varepsilon))}-\frac{P_{f}}{\mathrm{O}\left(P_{f}\right)}\right]_{\max } \\
& =\frac{1}{2 \mathrm{~N}^{2}-1}\left(P_{d}+1-2 P_{f}\right)_{\max } .
\end{aligned}
$$

Theorem 2: For CMD, the optimization $\lambda_{1}$ that maximizes $F=\frac{P_{d}}{O\left(P_{d}\right)}+\frac{1-P_{f}}{O(R(\tau, \varepsilon))}-\frac{P_{f}}{O\left(P_{f}\right)}$ consists in

$$
\begin{aligned}
& \lambda_{1}=\frac{-b+\sqrt{4 a c}}{2 a} \\
& \mathrm{a}=\left[(\mathrm{L}-1) \sqrt{\frac{2}{N_{s} \pi}}-\frac{\gamma}{\gamma+1} \lambda_{\mathrm{L}}\right] \cdot\left[2+(\mathrm{L}-1) \sqrt{\frac{2}{N_{s} \pi}}+\frac{\gamma}{\gamma+1} \lambda_{\mathrm{L}}\right] \\
& \mathrm{b}=\frac{4}{N_{s}} \ln \frac{2(\gamma+1)\left[1+(\mathrm{L}-1) \sqrt{2 / N_{s} \pi}\right]}{\gamma+1+\lambda_{L} \gamma} \\
& \mathrm{c}=2\left[(\mathrm{~L}-1) \sqrt{2 / N_{s} \pi}+\frac{\gamma}{\gamma+1} \lambda_{L}\right]
\end{aligned}
$$

The optimal sensing performance could be exhibited as

Proof: For CMD,

$$
F_{\max }=\frac{1}{L N_{s}}\left(P_{d}+1-2 P_{f}\right) \mid \varepsilon=\lambda_{1}
$$

$$
O\left(P_{d}\right)=O(R(\tau, \varepsilon))=\mathrm{O}\left(P_{f}\right) \approx L N_{s} .
$$

Based on (26)-(30) and (43), we can obtain

Due to

$$
\frac{\partial F}{\partial \lambda_{1}}=\frac{1}{L N_{s}}\left(\frac{\partial P_{d}}{\partial \lambda_{1}}-2 \frac{\partial P_{f}}{\partial \lambda_{1}}\right)
$$

$$
\begin{aligned}
& \frac{\partial P_{d}}{\partial \lambda_{1}}=\frac{\sqrt{\frac{N_{s}}{2}}\left(1+\frac{\gamma}{\gamma+1} \lambda_{L}\right)}{\sqrt{2 \pi} \lambda_{1}^{2}} \exp \left(-t_{2}^{2} / 2\right), \\
& \frac{\partial P_{f}}{\partial \lambda_{1}}=\frac{\sqrt{\frac{N_{s}}{2}}\left[1+\frac{2(\mathrm{~L}-1)}{N_{s} \pi}\right]}{\sqrt{2 \pi} \lambda_{1}^{2}} \exp \left(-t_{1}^{2} / 2\right), \\
& \mathrm{t}_{1}=\sqrt{N_{s} / 2}\left[1 / \lambda_{1}\left(1+(L-1) \sqrt{2 / N_{s} \pi}\right)-1\right], \\
& \mathrm{t}_{2}=\sqrt{N_{s} / 2}\left[1 / \lambda_{1}+\lambda_{L} \gamma / \lambda_{1}(\gamma+1)-1\right],
\end{aligned}
$$

then 
(59) could be further simplified as

$$
\frac{\partial F}{\partial \lambda_{1}} \leq 0 \Leftrightarrow \frac{\exp \left(-t_{2}^{2} / 2\right)}{\exp \left(-t_{1}^{2} / 2\right)} \leq \frac{2\left[1+(\mathrm{L}-1) \sqrt{\frac{2}{N_{s} \pi}}\right]}{1+\frac{\gamma}{\gamma+1} \lambda_{L}} .
$$

$$
\frac{\partial F}{\partial \lambda_{1}} \leq 0 \Leftrightarrow \frac{t_{1}^{2}}{2}-\frac{t_{2}^{2}}{2} \leq \ln \frac{2\left[1+(\mathrm{L}-1) \sqrt{\frac{2}{N_{s} \pi}}\right]}{1+\frac{\gamma}{\gamma+1} \lambda_{L}}
$$

$\frac{t_{1}^{2}}{2}-\frac{t_{2}^{2}}{2}=\ln \frac{2\left[1+(\mathrm{L}-1) \sqrt{\frac{2}{N_{s} \pi}}\right]}{1+\frac{\gamma}{\gamma+1} \lambda_{L}}$ is the function on $\lambda_{1}$. By solving this linear equation in two unknowns, the conclusion in Theorem 2 can be reached.

\subsection{Extensions to CSS}

Theorem 3: For AND fusion rule-based CSS, the sensing performance is optimal when

$$
\varepsilon=\varepsilon_{2}=\left[\frac{1}{2}+\sqrt{\frac{1}{4}+\frac{\gamma}{2}+\frac{2 \gamma+1}{\gamma \sqrt{N}} \ln 2 \sqrt{2 \gamma+1}}\right] \sigma_{n}^{2},
$$

and the corresponding performance could be formulated as

$$
F_{\text {max }}=\frac{\left(P_{d}\right)^{K}+1-2\left(P_{f}\right)^{K}}{2 K N+2 K} \mid \varepsilon=\varepsilon_{2} .
$$

Proof: The proof of this theorem can refer to the demonstration in Theorem 2.

Note that for AND fusion rule-based CSS,

$$
O\left(P_{d}(\mathrm{~N}, \varepsilon)\right)=O(R(\tau, \varepsilon))=\mathrm{O}\left(P_{f}(\mathrm{~N}, \varepsilon)\right)=2 K N+2 K
$$

Theorem 4: As for OR fusion rule-based CSS, the optimal sensing performance arrives as

$$
\varepsilon=\left[\frac{1}{2}+\sqrt{\frac{\gamma}{2}+\frac{1}{4}+\frac{(2 \gamma+1) \ln 2}{N \gamma}+\frac{(2 \gamma+1)(\mathrm{K}+1) \ln \sqrt{2 \gamma+1}}{2 N \gamma}}\right] \sigma_{n}^{2} .(66)
$$

and its sensing performance could be written as

$$
F_{\max }=\frac{2\left(1-\mathrm{P}_{f}\right)^{K}-\left(1-\mathrm{P}_{d}\right)^{K}}{2 K N+2 K} .
$$

Proof: For OR fusion rule-based CSS,

$$
O\left(P_{d}(\mathrm{~N}, \varepsilon)\right)=O(R(\tau, \varepsilon))=\mathrm{O}\left(P_{f}(\mathrm{~N}, \varepsilon)\right)=2 K N+2 K .
$$

On the basis of (6)-(8) and (33)-(34),

then

$$
\frac{\partial F}{\partial \varepsilon}=K\left(1-\mathrm{P}_{d}\right)^{K-1} \frac{\partial \mathrm{P}_{d}}{\partial \varepsilon}-2 K\left(1-\mathrm{P}_{f}\right)^{K-1} \frac{\partial \mathrm{P}_{f}}{\partial \varepsilon},
$$

$$
\frac{\partial F}{\partial \varepsilon} \leq 0 \Leftrightarrow \frac{\partial \mathrm{P}_{d}}{\partial \varepsilon} / \frac{\partial \mathrm{P}_{f} \leq}{\partial \varepsilon} 2\left(\frac{1-\mathrm{P}_{f}}{1-\mathrm{P}_{d}}\right)^{K-1}
$$


By reason of

$$
\frac{1-\mathrm{P}_{f}}{1-\mathrm{P}_{d}} \approx \sqrt{2 \gamma+1}
$$

(69) could be rewritten as

$$
\begin{gathered}
\frac{\partial F \geq 0 \Leftrightarrow \exp \left(x_{1}-x_{2}\right) \leq 2 \ln 2 \sqrt{2 \gamma+1}+(\mathrm{K}-1) \ln \sqrt{2 \gamma+1}}{\partial \varepsilon \leq} \\
x_{1}=\frac{1}{2}\left[\left(\frac{\varepsilon}{\sigma_{n}^{2}}-1\right) \sqrt{\tau f_{s}}\right]^{2} \\
x_{2}=\frac{1}{2}\left[\left(\frac{\varepsilon}{\sigma_{n}^{2}}-\gamma-1\right) \sqrt{\frac{\tau f_{s}}{2 \gamma+1}}\right]^{2} .
\end{gathered}
$$

(72) could be further simplified as

$$
2\left(\frac{\varepsilon}{\sigma_{n}^{2}}-\frac{1}{2}\right)^{2}-\gamma-\frac{1}{2} \leq \frac{(2 \gamma+1) \ln 2}{N \gamma}+\frac{(2 \gamma+1)(\mathrm{K}+1) \ln \sqrt{2 \gamma+1}}{2 N \gamma} .
$$

That is

$$
\varepsilon_{\leq}\left[\frac{1}{2}+\sqrt{\frac{\gamma}{2}+\frac{1}{4}+\frac{(2 \gamma+1) \ln 2}{N \gamma}+\frac{(2 \gamma+1)(\mathrm{K}+1) \ln \sqrt{2 \gamma+1}}{2 N \gamma}}\right] \sigma_{n}^{2} .
$$

Theorem 5: With regard to N-out-of-K fusion rule-based CSS, the optimal threshold $\left(\varepsilon=\varepsilon^{*}\right)$ exists that maximizes the sensing strategy in (41).

Proof: Based on (6)-(8) and (35)-(36), we obtain

$$
\begin{aligned}
& \frac{\partial P_{d}(N, \varepsilon)}{\partial \varepsilon}=\frac{\partial P_{d}}{\partial \varepsilon} \sum_{i=N}^{K}\left(\begin{array}{c}
K \\
i
\end{array}\right) P_{d}^{i-1}\left(1-\mathrm{P}_{d}\right)^{K-i}\left[\mathrm{i}-(\mathrm{K}-\mathrm{i}) \frac{P_{d}}{1-P_{d}}\right] \\
& \frac{\partial P_{f}(N, \varepsilon)}{\partial \varepsilon}=\frac{\partial P_{f}}{\partial \varepsilon} \sum_{i=N}^{K}\left(\begin{array}{c}
K \\
i
\end{array}\right) P_{f}^{i-1}\left(1-\mathrm{P}_{f}\right)^{K-i}\left[\mathrm{i}-(\mathrm{K}-\mathrm{i}) \frac{P_{f}}{1-P_{f}}\right] .
\end{aligned}
$$

From (41), we have

$$
\frac{\partial F}{\partial \varepsilon} \leq 0 \Leftrightarrow \frac{\partial P_{d}(N, \varepsilon)}{\partial \varepsilon} / \frac{\partial P_{f}(N, \varepsilon) \leq}{\partial \varepsilon} \leq .
$$

According to (76)-(78), we can get

$$
\frac{\partial F}{\partial \varepsilon} \leq 0 \Leftrightarrow \frac{\partial P_{d}}{\partial \varepsilon} / \frac{\partial P_{f}}{\partial \varepsilon} \sum_{i=N}^{K}\left(\begin{array}{c}
K \\
i
\end{array}\right)\left(\frac{P_{d}}{P_{f}}\right)^{i-1}\left(\frac{1-\mathrm{P}_{d}}{1-\mathrm{P}_{f}}\right)^{K-i} \frac{\mathrm{i}-(\mathrm{K}-\mathrm{i}) \frac{P_{d}}{1-P_{d}} \geq}{\mathrm{i}-(\mathrm{K}-\mathrm{i}) \frac{P_{f}}{1-P_{f}}} \leq 2 .
$$

The solution to (80) can be evaluated numerically ( $\varepsilon=\varepsilon^{*}$ ). The solution is exactly the optimal detection threshold. Then the optimal sensing performance could be denoted as

$$
\mathrm{F}_{\max }=\frac{P_{d}(N, \varepsilon)+1-2 P_{f}(N, \varepsilon)}{2 K N+2 K} \mid \varepsilon=\varepsilon^{*} .
$$

Fig. 1 exhibits the flow chart of the proposed sensing strategy. Firstly, four efficiency functions $\left(\eta_{d}, \eta_{f}, \eta_{R}\right.$ and $\eta$ ) are defined to describe sensing efficiency of spectrum sensing schemes (ED, CFD, CMD and CSS), where $\eta_{d}$ denotes efficiency function of a CR to protect 
PU, $\eta_{f}$ expresses efficiency function of a false alarm, $\eta_{R}$ shows communication efficiency of secondary network and $\eta$ remarks the overall efficiency function of a spectrum sensing scheme. Then given sensing capacity and sensing complexity, the optimal sensing strategy is obtained based on the maximization of the overall efficiency function $\eta$. Finally, $\eta$ is applied to classical spectrum sensing schemes and the corresponding optimal sensing performance is obtained and demonstrated therein.

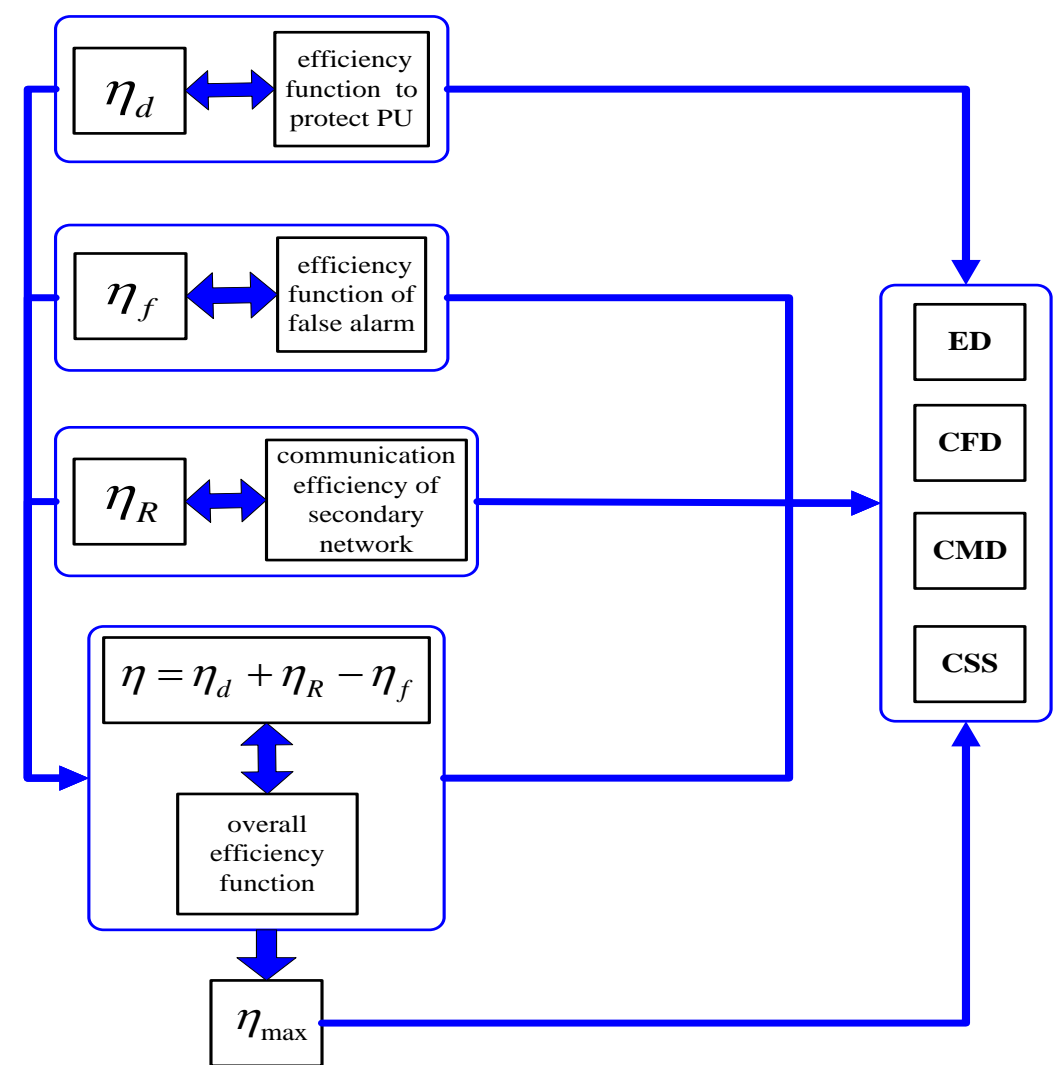

Fig. 1. The flow chart of the proposed sensing strategy

\section{Simulation and discussion}

In this section, simulations based on MATLAB platform are made to examine the performance of the proposed efficiency functions in (37)-(39) and the novel sensing strategies in (43). Orthogonal Frequency Division Multiplexing (OFDM) signal [32] is chosen as the test signal (PU) for its generality and popularity in wireless communication networks, whose center frequency is denoted as $f_{c}=1.0 \times 10^{8} \mathrm{hz}$ and sampling frequency is denoted as $f_{s}=3.0 \times 10^{8} \mathrm{hz}$. The sampling point at the receiving terminal of a CR is $N=f_{s} \tau=1000$ without special instructions. The sensing performance is obtained under various signal-noise-ratio (SNR) in AWGN, Rayleigh and Rician channel, respectively. The Doppler shift in both Rayleigh and Rician channel is set as 250, the Rice factor in the Rician channel is fixed as $K=3$. In addition, the simulation values are magnified 1000 times in amplitude for convenience. 


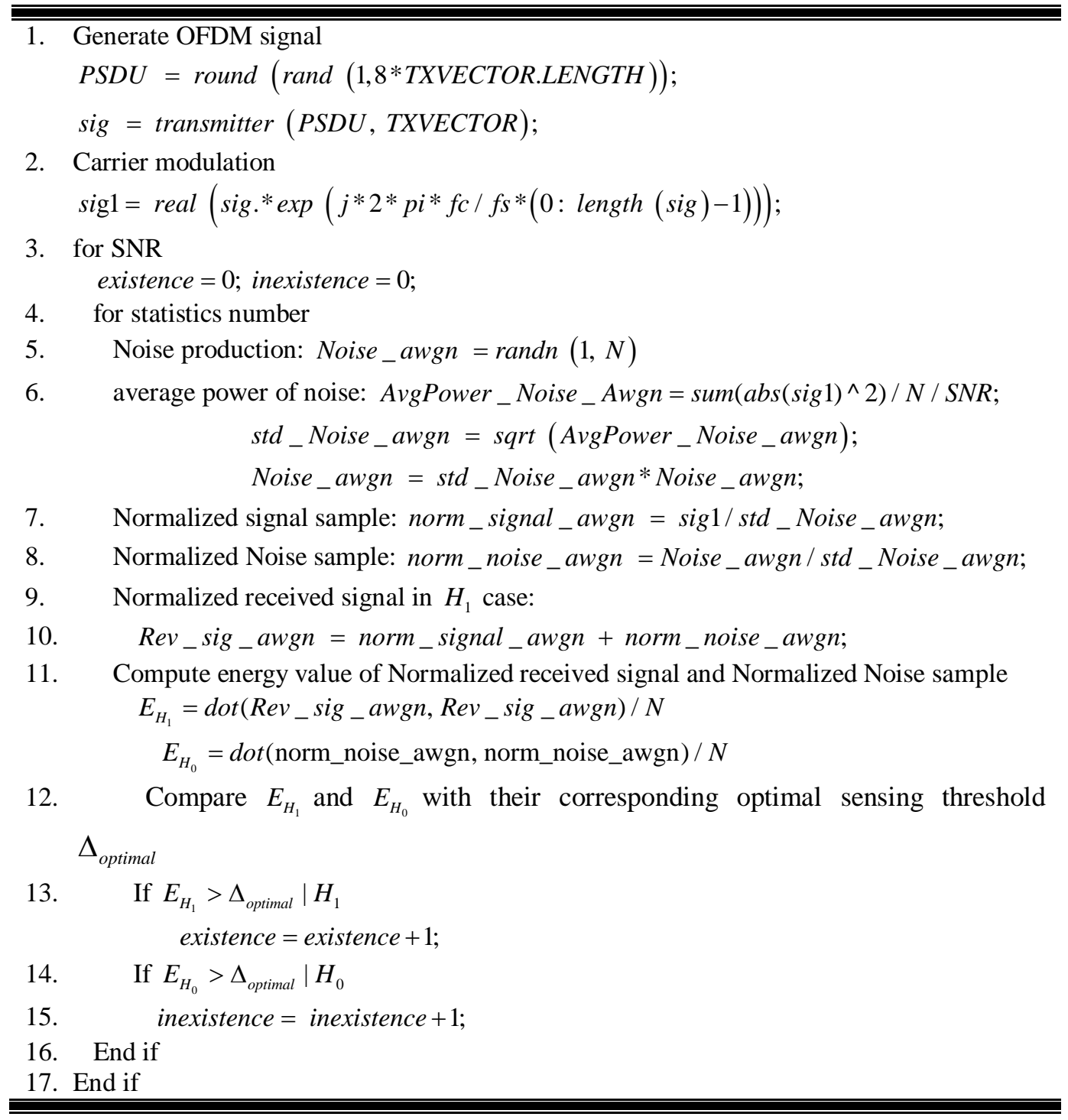

Fig. 2. Coding flow of the proposed sensing strategy

Fig. 2 shows coding flow of the proposed sensing strategy. It is noted that Fig. 2 takes AWGN channel for example, where PSDU denotes the generated random number, TXVECTOR.LENGTH $=2000$ and TXVECTOR.DATARATE $=6 . \quad$ In addition, $f_{c}=1.0 \times 10^{8} \mathrm{hz}$ denotes the carrier frequency and $f_{s}=3.0 \times 10^{8} \mathrm{hz}$ denotes the sampling frequency at the receiver of CR.

Fig. 3 gives $\eta_{f}$ comparisons of various schemes under different SNRs in various channels when $\eta_{\max }$ is maximum. The $\eta_{f}$ of ED in AWGN, Rayleigh and Rician channel are all zero. Consequently, there is only one label in Fig. 3. So is Fig. 4 or Fig. 5. From Fig. 3, ED has the lowest $\eta_{f}$ score and CFD takes second place while CMD has the highest $\eta_{f}$ score under equal conditions. This is due to that the energy value of received signal is more sensitive than its corresponding Cyclostationary feature and correlation for spectrum sensing given sensing 
capacity and sensing complexity, which indicates that ED has the highest efficiency to discover idle spectrum while this capacity of CMD is the worst.

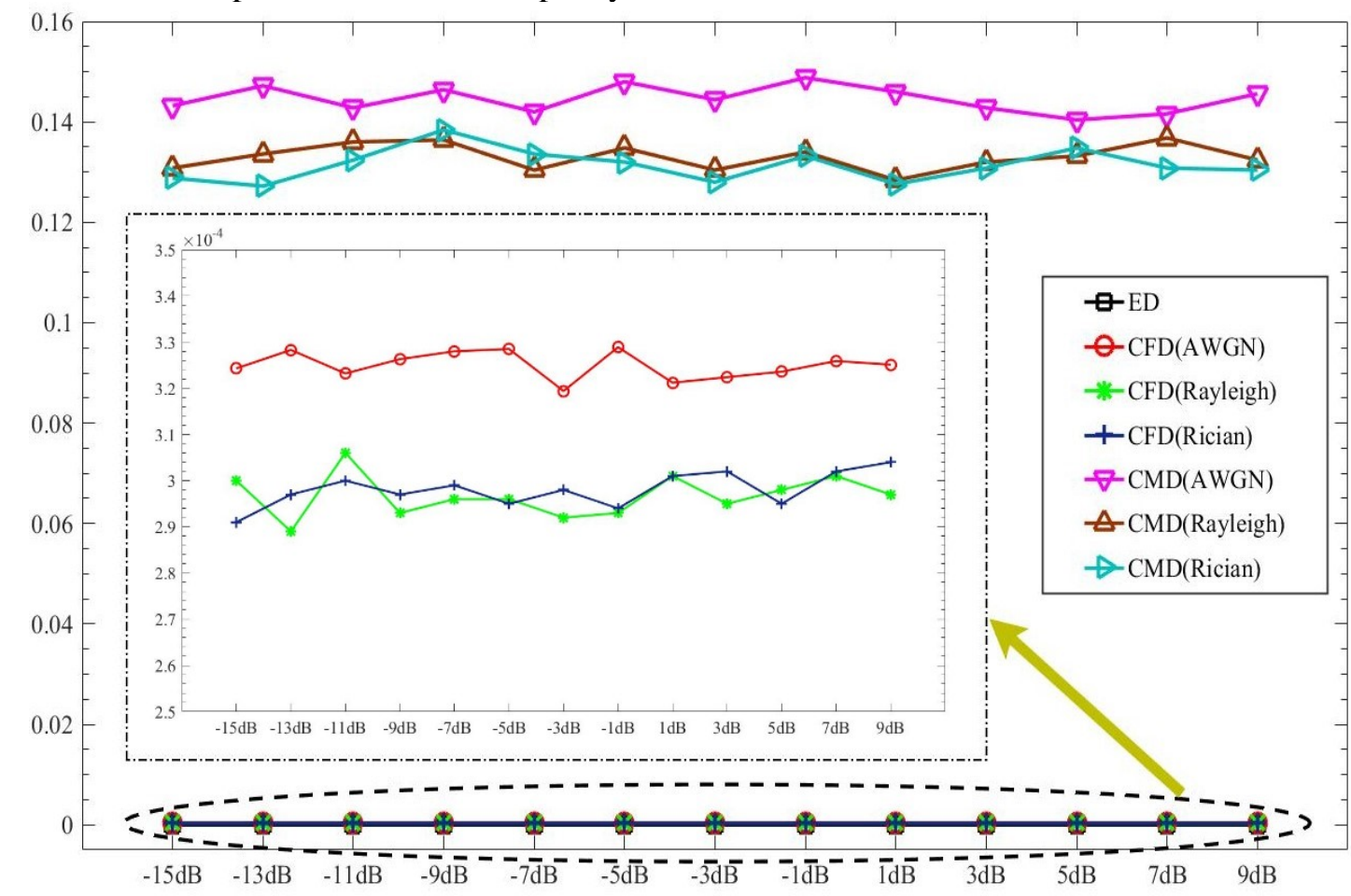

Fig. 3. $\eta_{f}$ comparisons of various schemes under different scenarios

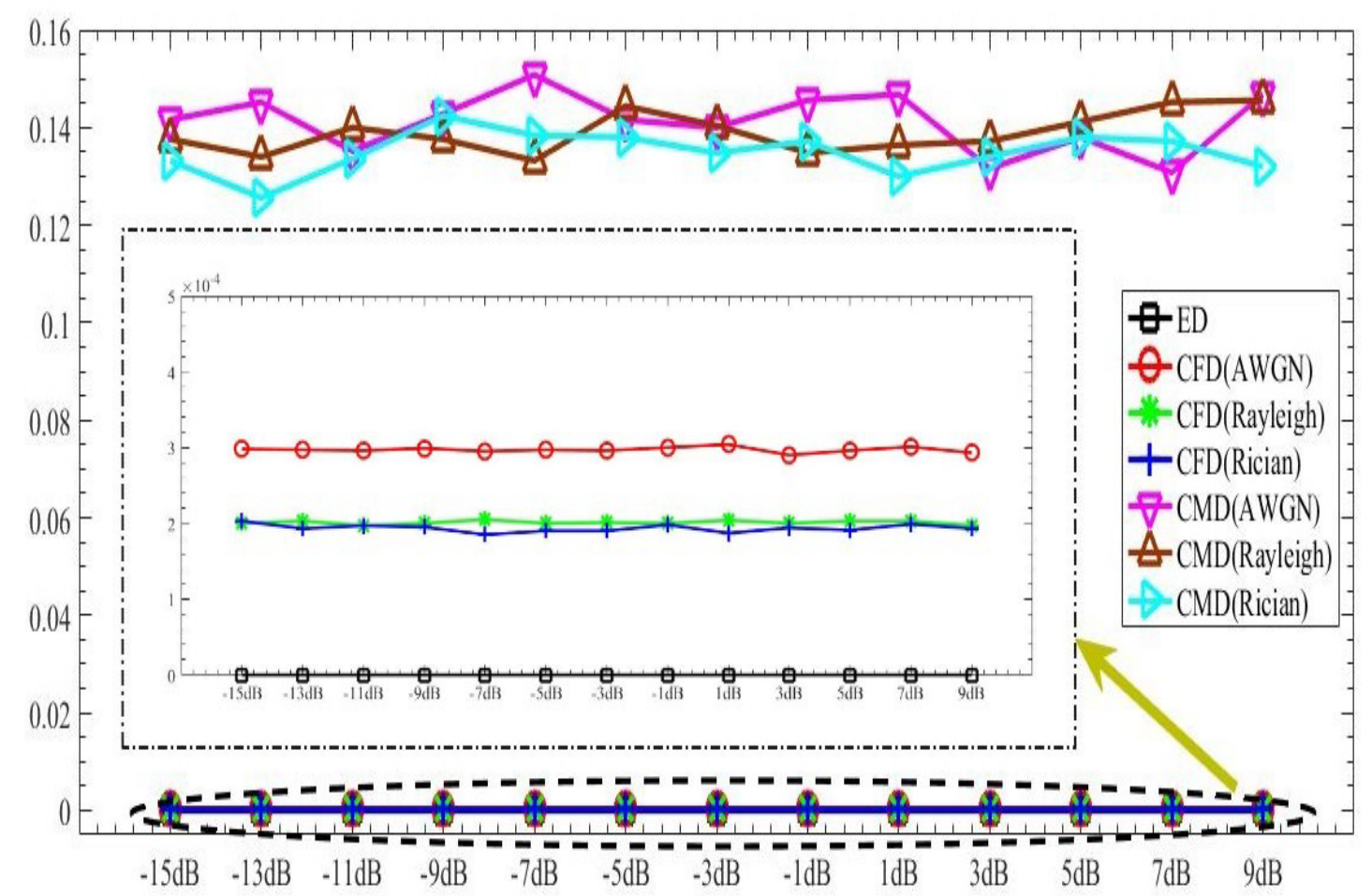

Fig. 4. $\eta_{d}$ comparisons of various schemes under different scenarios 


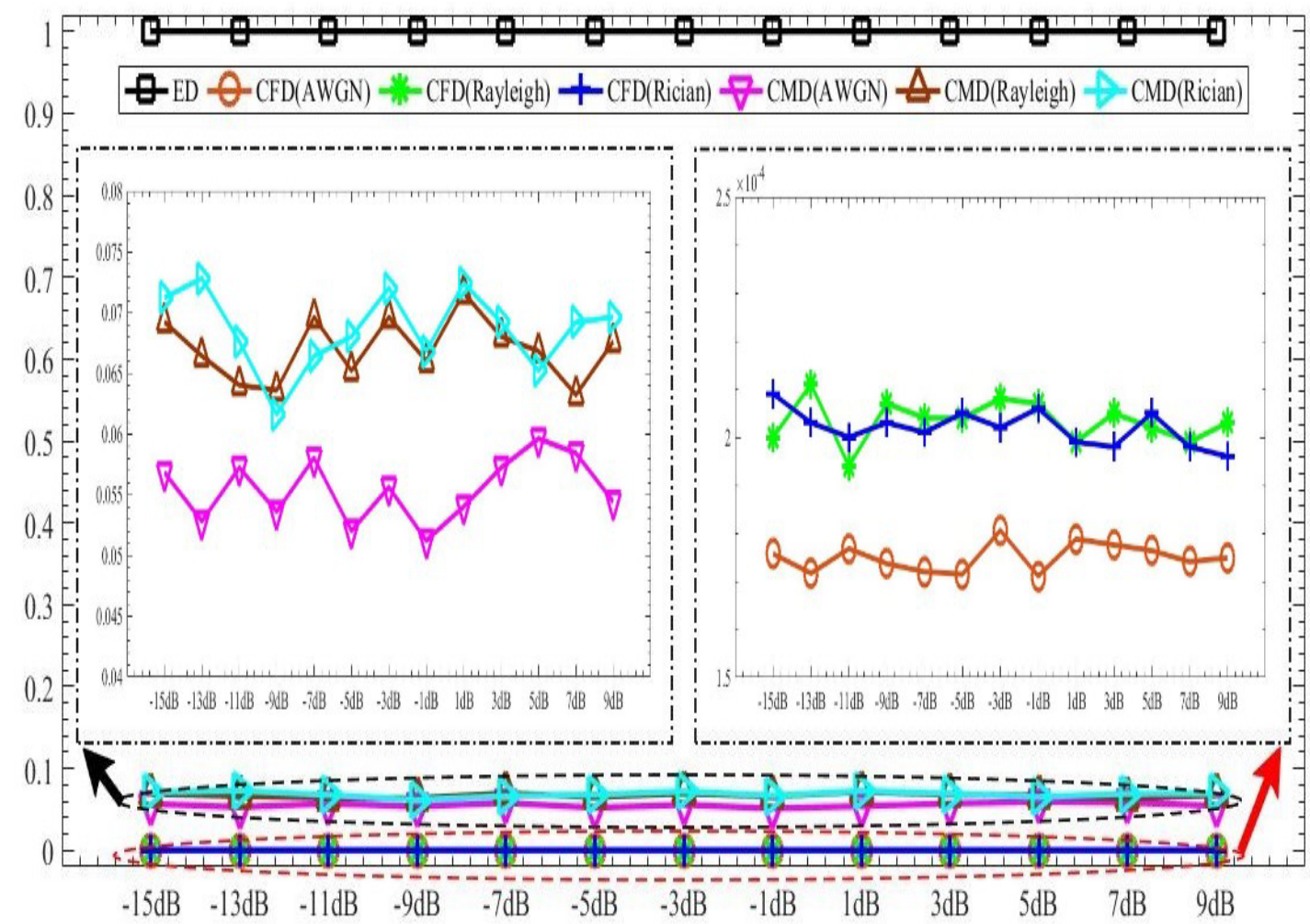

Fig. 5. $\eta_{R}$ comparisons of various schemes under different scenarios

$\eta_{d}$ comparisons of various schemes are made under different scenarios in Fig. 4 when sensing performance is optimal according to (45), (49) and (54). In contrast to the case in Fig. 3 , the $\eta_{d}$ of ED is the worst while CMD has the highest $\eta_{d}$ score. The reason for these is that ED suffers from the influence of noise uncertainty. It signifies that CMD has the highest efficiency to protect PU from possible interference of SU and this capacity of ED is inferior compared with the others.

Fig. 5 provides $\eta_{R}$ comparisons for ED, CFD and CMD under various scenarios when sensing performance is optimal according to (45), (49) and (54). For convenience, $C_{0} P\left(H_{0}\right)\left(1-\frac{\tau}{T}\right)$ is regarded as 1 in the same condition for ED, CFD and CMD. Thus, Fig. 5 works as a supplement of Fig. 3. From Fig. 5, the $\eta_{R}$ of ED is the highest, which manifests the superior communication efficiency of ED with its users. Compared with ED, the $\eta_{R}$ of CMD and CFD declines sharply. It states clearly that the higher protection efficiency of CMD and CFD for PU is at the expense of their communication efficiency with corresponding users.

To sum up, the proposed efficiency functions in (37)-(39) offer deep insight into the sensing performance of different spectrum sensing scheme given false alarm probability, detection probability, available throughput and computational complexity. On this basis, some significant conclusions are drawn.:

1) In the respect of communication efficiency in secondary networks, ED is the optimal selection.

2) CFD and CMM are adept at protecting PU from possible interference of SU. 
3) Multistage detection given the combination of ED and CFD (or CMM) may consider both communication efficiency of secondary networks and PU protection.

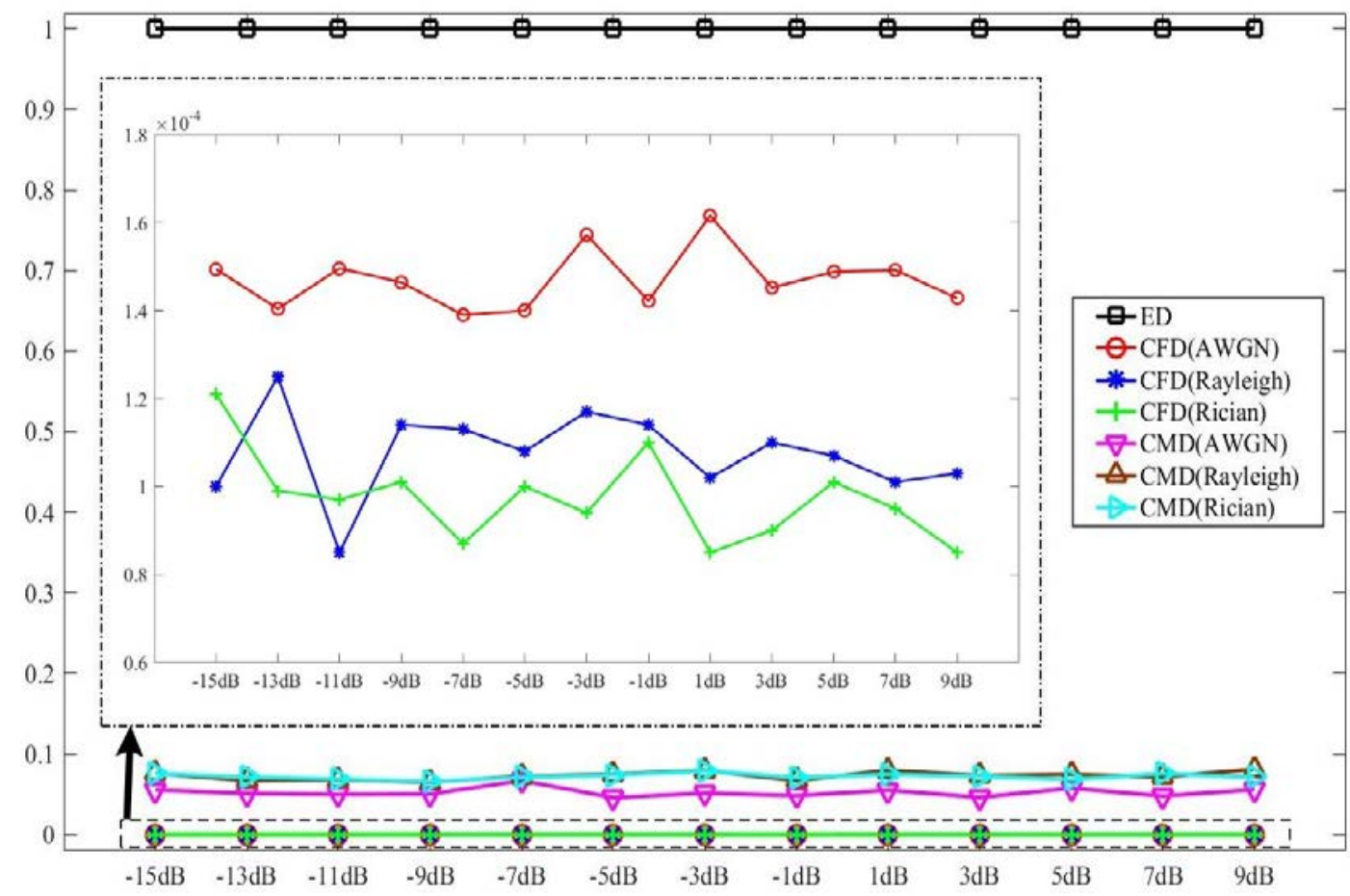

Fig. 6. $\eta_{\max }$ comparisons of various schemes under different scenarios

Fig. 6 exhibits $\eta_{\max }$ comparisons of various schemes under different scenarios. From Fig. 6, ED is a SS scheme with the highest efficiency overall; CMD takes the second place while CFD has the lowest efficiency within the compared schemes. This indicates that ED is the optimal selection for SS in terms of both PU protection and communication capacity in secondary network. The computational complexity of CMD and CFD are relatively higher than ED, therefore their efficiency is lower than ED.

In summary, the sensing strategy in (43) provides a new perspective to estimate various schemes of SS, where both sensing performance and computation complexity is considered. As a result, this works as a global sensing strategy that considers the whole primary factors that influence sensing performance and gives a superior majorization selection for SS.

\section{Conclusions}

In this paper, three efficiency functions are provided to estimate the sensing efficiency of a spectrum sensing scheme given sensing performance and computational complexity. On this basis, a novel sensing strategy is proposed and demonstrated to detect idle spectrum with a higher efficiency. Furthermore, the proposed sensing strategy is applied to energy detector, Cyclostationary feature detector, covariance matrix detector and cooperative spectrum detector. Some significant conclusions are reached therein. Moreover, simulations also validate the effectiveness of the proposed scheme. 


\section{References}

[1] Amjad M, Rehmani M H, Mao S, "Wireless Multimedia Cognitive Radio Networks: A Comprehensive Survey,” IEEE Communications Surveys \& Tutorials, vol. 20, no. 2, pp. 1056-1103, 2018. Article (CrossRef Link)

[2] Khan A A, Rehmani M H, Rachedi A, "Cognitive-Radio-Based Internet of Things: Applications, Architectures, Spectrum Related Functionalities, and Future Research Directions,” IEEE Wireless Communications, 24(3), 17-25, 2017. Article (CrossRef Link)

[3] Haykin S, "Cognitive radio: brain-empowered wireless communications," IEEE Journal on Selected Areas in Communications, 23(2), 201-220, 2005. Article (CrossRef Link)

[4] Mitola J I, Maguire G Q J, “Cognitive radio: making software radios more personal,” IEEE Pers Commun, 6(4), 13-18, 1999. Article (CrossRef Link)

[5] Wang Q, Dai H N, Georgiou O, et al., "Connectivity of Underlay Cognitive Radio Networks with Directional Antennas,” IEEE Transactions on Vehicular Technology, vol. 67, no. 8, pp. 7003-7017, 2018. Article (CrossRef Link)

[6] Lv L, Yang L, Jiang H, et al., "When NOMA Meets Multiuser Cognitive Radio: Opportunistic Cooperation and User Scheduling,” IEEE Transactions on Vehicular Technology, vol. 67, no. 7, pp. 6679-6684, 2018,. Article (CrossRef Link)

[7] Bishnu A, Bhatia V, "LogDet Covariance Based Spectrum Sensing under Colored Noise,” IEEE Transactions on Vehicular Technology, vol. 67, no. 7, pp. 6716-6720, 2018. Article (CrossRef Link)

[8] Qi P, Li Z, Li H, et al., "Blind Sub-Nyquist Spectrum Sensing with Modulated Wideband Converter,” IEEE Transactions on Vehicular Technology, vol. 67, no. 5, pp. 4278-4288, 2018. Article (CrossRef Link)

[9] Khanikar K, Sinha R, Bhattacharjee R, “Cooperative Spectrum Sensing using Quantized Energy Statistics in the Absence of Dedicated Reporting Channel," IEEE Transactions on Vehicular Technology, vol. 67, no. 5, pp. 4149-4160, 2018. Article (CrossRef Link)

[10] Bera D, Chakrabarti I, Pathak S S, et al., “Another Look in the Analysis of Cooperative Spectrum Sensing over Nakagami- \$m\$ Fading Channels,” IEEE Transactions on Wireless Communications, 16(2), 856-871, 2017. Article (CrossRef Link)

[11] Liang Y C, Zeng Y, Peh E C Y, et al., "Sensing-Throughput Tradeoff for Cognitive Radio Networks,” in Proc. of 2007 IEEE International Conference on Communications, 2007. Article (CrossRef Link)

[12] Mu J, Jing X, Huang H, et al., “Joint Spectrum Sensing Based on Variance and Correlation Analysis,” China communications, 14(10), 219-227, 2017.

[13] Mu J, Jing X, Huang H, et al., "Subspace based method for spectrum sensing with multiple users over fading channel,” IEEE Communications Letters, vol. 22, pp. 848-851, 2018. Article (CrossRef Link)

[14] Digham F F, Alouini M S, Simon M K, “On the Energy Detection of Unknown Signals Over Fading Channels,” IEEE Transactions on Communications, 55(1), 21-24, 2007. Article (CrossRef Link)

[15] Shen J C, Alsusa E, "Joint Cycle Frequencies and Lags Utilization in Cyclostationary Feature Spectrum Sensing,” IEEE Transactions on Signal Processing, 61(21), 5337-5346, 2013. Article (CrossRef Link)

[16] Jin M, Guo Q, Xi J, et al., "Spectrum Sensing Using Weighted Covariance Matrix in Rayleigh Fading Channels,” IEEE Transactions on Vehicular Technology, 64(11), 5137-5148, 2015. Article (CrossRef Link)

[17] Ma J, Zhao G, Li Y, "Soft Combination and Detection for Cooperative Spectrum Sensing in Cognitive Radio Networks,” IEEE Transactions on Wireless Communications, 7(11), 4502-4507, 2008. Article (CrossRef Link)

[18] Digham F F, Alouini M S, Simon M K, “On the Energy Detection of Unknown Signals Over Fading Channels,” IEEE Transactions on Communications, 55(1), 21-24, 2007. Article (CrossRef Link) 
[19] Atapattu S, Tellambura C, Jiang H, “Analysis of area under the ROC curve of energy detection,” IEEE Transactions on Wireless Communications, 9(3), 1216-1225, 2010. Article (CrossRef Link)

[20] Peh E C Y, Liang Y C, Guan Y L, “Optimization of Cooperative Sensing in Cognitive Radio Networks: A Sensing-Throughput Tradeoff View,” IEEE Transactions on Vehicular Technology, vol. 58, pp. 5294-5299, 2009. Article (CrossRef Link)

[21] Enserink S, Cochran D, “A cyclostationary feature detector,” in Proc. of Signals, Systems and Computers, 1994. 1994 Conference Record of the Twenty-Eighth Asilomar Conference on. IEEE, 1994. Article (CrossRef Link)

[22] Turunen V, Kosunen M, Huttunen A, et al., "Implementation of Cyclostationary Feature Detector for Cognitive Radios,” in Proc. of International Conference on Cognitive Radio Oriented Wireless Networks and Communications, Crowncom. IEEE, 1-4, 2009. Article (CrossRef Link)

[23] Choi K W, Jeon W S, Dong G J, "Sequential detection of cyclostationary signal for cognitive radio systems,” IEEE Transactions on Wireless Communications, 8(9), 4480-4485, 2009. Article (CrossRef Link)

[24] Yang M, Li Y, Liu X, et al., "Cyclostationary Feature Detection Based Spectrum Sensing Algorithm under Complicated Electromagnetic Environment in Cognitive Radio Networks,” China communications, 12(9), 35-44, 2015. Article (CrossRef Link)

[25] Satija U, Ramkumar B, Manikandan M S, "A Novel Sparse Classifier for Automatic Modulation Classification using Cyclostationary Features," Wireless Personal Communications, 96(3), 4895-4917, 2017. Article (CrossRef Link)

[26] Du K L, Mow W H, “Affordable Cyclostationarity-Based Spectrum Sensing for Cognitive Radio with Smart Antennas,” IEEE Transactions on Vehicular Technology, 59(4), 1877-1886, 2010. Article (CrossRef Link)

[27] S. Kozłowski, "Implementation and verification of cyclostationary feature detector for DVB-T signals,” Iet Signal Processing, 10(2), 162-167, 2016. Article (CrossRef Link)

[28] Zeng Y, Liang Y C, "Spectrum-Sensing Algorithms for Cognitive Radio Based on Statistical Covariances,” IEEE Transactions on Vehicular Technology, 58(4), 1804-1815, 2008. Article (CrossRef Link)

[29] Akyildiz I F, Lo B F, Balakrishnan R, “Cooperative spectrum sensing in cognitive radio networks: A survey,” Physical Communication, 4(1), 40-62, 2011. Article (CrossRef Link)

[30] Letaief K B, Zhang W, “Cooperative Spectrum Sensing,” Cognitive Wireless Communication Networks. Springer US, 115-138, 2007.

[31] Ganesan G, Li Y, "Cooperative Spectrum Sensing in Cognitive Radio, Part I: Two User Networks,” Wireless Communications IEEE Transactions on, 6(6), 2204-2213, 2007. Article (CrossRef Link)

[32] Hwang C H, Lai G L, Chen S C, “Spectrum Sensing in Wideband OFDM Cognitive Radios,” IEEE Transactions on Signal Processing, 58(2), 709-719, 2010. Article (CrossRef Link) 


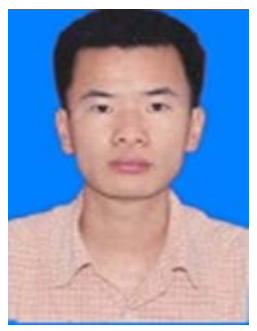

Huang Kewen (1980 -), male, Master of Software Engineering, graduated from Huazhong University of Science and Technology, Experimenter. His research mainly focuses on physics, electronics and communication technology.

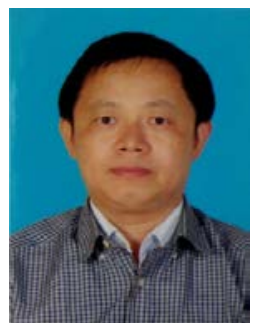

Liu Yimin (1965 -), male, Doctor, graduated from Sun Yat-sen University, Professor. He is mainly engaged in physics teaching and laboratory management research.

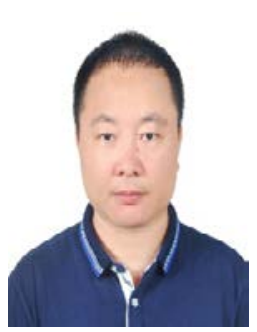

Hong Yuanquan received the B.S. degree in electronic information science and technology and the M.S. degree in circuit and system from the South China Normal University, Guangzhou, China, in 2003 and 2009 respectively. He is currently a senior experimentalist with the School of Physics and Electromechanical Engineering, Shaoguan University, China. His research interests include cognitive radio, signal processing, beamforming/precoding using large antenna arrays, and physical layer security in millimeter-wave wireless.

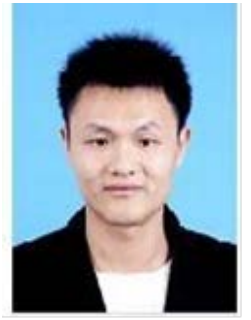

Junsheng Mu received B.S. and M.S. degrees in 2012 and respectively, and he is currently working towards the Ph.D. degree in Information and Communication Engineering at Beijing University of Posts and Telecommunications. His research interests include spectrum sensing and pattern recognition. 